\title{
Astrocytic Actions on Extrasynaptic Neuronal Currents
}

\author{
Balázs Pál * \\ Department of Physiology, Faculty of Medicine, University of Debrecen, Debrecen, Hungary
}

In the last few decades, knowledge about astrocytic functions has significantly increased. It was demonstrated that astrocytes are not passive elements of the central nervous system (CNS), but active partners of neurons. There is a growing body of knowledge about the calcium excitability of astrocytes, the actions of different gliotransmitters and their release mechanisms, as well as the participation of astrocytes in the regulation of synaptic functions and their contribution to synaptic plasticity. However, astrocytic functions are even more complex than being a partner of the "tripartite synapse," as they can influence extrasynaptic neuronal currents either by releasing substances or regulating ambient neurotransmitter levels. Several types of currents or changes of membrane potential with different kinetics and via different mechanisms can be elicited by astrocytic activity. Astrocyte-dependent phasic or tonic, inward or outward currents were described in several brain areas. Such currents, together with the synaptic actions of astrocytes, can contribute to neuromodulatory mechanisms, neurosensory and -secretory processes, cortical oscillatory activity, memory, and learning or overall neuronal excitability. This mini-review is an attempt to give a brief summary of astrocyte-dependent extrasynaptic neuronal currents and their possible functional significance.

Keywords: astrocyte-neuron interactions, slow inward current, slow outward current, tonic current, gliotransmitters

\section{OPEN ACCESS}

Edited by:

Alessandro Tozzi,

University of Perugia, Italy

Reviewed by:

C. Peter Bengtson,

University of Heidelberg, Germany

László Héja,

Hungarian Academy of Sciences,

Hungary

*Correspondence:

Balázs Pál

pal.balazs@med.unideb.hu

Received: 10 September 2015 Accepted: 20 November 2015 Published: 09 December 2015

Citation:

Pál B (2015) Astrocytic Actions on Extrasynaptic Neuronal Currents.

Front. Cell. Neurosci. 9:474. doi: 10.3389/fncel.2015.00474
Abbreviations: 7-Cl-KYN, 7-chlorokynurenate; $\alpha$-AA, alpha-aminoadipic acid; ACEA, arachidonyl-2'-chloroethylamide ( $N$-(2-chloroethyl)-5Z,8Z,11Z,14Z-eicosatetraenamide); ACSF, artificial cerebrospinal fluid; ATP, adenosine triphosphate; BAPTA, 1,2-bis(o-aminophenoxy)ethane-N,N,N'N'-tetraacetic acid; BBG, Brilliant Blue G; BzATP, $2^{\prime}\left(3^{\prime}\right)-O-(4-$ benzoylbenzoyl)adenosine- $5^{\prime}$-triphosphate; $\mathrm{CB} 1$ receptor, cannabinoid type 1 receptor; CNS, central nervous system; CPCCOEt, 7-(hydroxyimino)cyclopropa[ $b]$ chromen-1a-carboxylate ethyl ester; DAAO, D-amino acid oxidase; D-AP5, D-(-)-2-amino-5-phosphonopentanoic acid; DHPG, 3,5-dihydroxyphenylglycine; DIDS, 4,4'diisothiocyanato-2,2'-stilbenedisulfonic acid; DG, dentate gyrus; EAAT1-4, excitatory amino acid transporter type 1-4; EPSC, excitatory postsynaptic current; GABA, $\gamma$-amino butyric acid; GAT2-3, GABA transporter type 2-3; GLYT1, glycine transporter type 1; GYKI53655, 1-(4-aminophenyl)-3-methylcarbamyl-4-methyl-3,4-dihydro7,8-methylenedioxy-5H-2,3-benzodiazepine; Il-1, interleukin-1; IPSC, inhibitory postsynaptic current; LY341495, (2S)-2-amino-2-[(1S,2S)-2-carboxycycloprop-1-yl]-3-(xanth-9-yl) propanoic acid; mGluR, metabotropic glutamate receptor; MK801, (5R,10S)-(-)-5-methyl-10,11-dihydro-5H-dibenzo[ $[a, d]$ cylcohepten-5,10-imine; MSO, methionine sulfoximine; NBQX, 2,3-dioxo-6-nitro-1,2,3,4-tetrahydrobenzo[f]quinoxaline-7-sulfonamide; NMDA, N-methylD-aspartate; NP-EGTA, o-nitrophenyl-ethylene glycol-bis(2-aminoethylether)- $N, N, N, N^{\prime}$-tetraacetic acid; NPPB, 5-nitro-2-(3-phenylpropylamino)benzoic acid; NR2B, NMDA receptor subunit 2B; NVP-AAM077, [(S)-\{[(1S)-1-(4bromophenyl)ethyl]amino\}(2,3-dioxo-1,2,3,4-tetrahydroquinoxalin-5-yl)methyl]phosphonic acid; ORG24598, $N$-methyl$N$-[(3R)-3-phenyl-3-[4-(trifluoromethyl)phenoxy]propyl]-glycine; ORG25543, $N$-[[1-(dimethylamino)cyclopentyl]methyl]3,5-dimethoxy-4-(phenylmethoxy)benzamide; OxATP, oxidized adenosine triphosphate; $\mathrm{P} 2 \mathrm{X}_{7}$, purinergic receptor type $2 \mathrm{X}_{7}$; $\mathrm{P} 2 \mathrm{X}_{4}$, purinergic receptor type $2 \mathrm{X}_{4} ; \mathrm{P}_{2} \mathrm{Y}_{1}$, purinergic receptor type $2 \mathrm{Y}_{1}$; PAR-1, protease-activated receptor type 1; PGE2, prostaglandine E2; PPDA, $\left(2 S^{*}, 3 R^{*}\right)$-1-(phenanthren-2-carbonyl)piperazine-2,3-dicarboxylic acid; Ro25-6981, $(\alpha R, \beta S)$ - $\alpha$ (4-hydroxyphenyl)- $\beta$-methyl-4-(phenylmethyl)-1-piperidinepropanol; SERCA, sarco/endoplasmic reticulum $\mathrm{Ca}^{2+}$-ATPase; SIC, slow inward current; SOC, slow outward current; t-ACPD, $( \pm)$-1-aminocyclopentane-trans-1,3-dicarboxylic acid; TBOA, threo- $\beta$-benzyloxyaspartic acid; TFLLR-NH2, Thr-Phe-Leu-Leu-Arg- $\mathrm{NH}_{2}$ (PAR1 agonist); TNF $\alpha$, tumor necrosis factor $\alpha$; TTX, tetrodotoxin; VRAC, volume-regulated anionic channel. 


\section{INTRODUCTION}

It was extensively demonstrated in the last decades that glial cells, especially astrocytes are not passive elements of the brain, but active partners of the neurons in signal processing (Araque et al., 1999, 2014; Perea et al., 2009). Astrocytes are known as non-excitable cells, as they can't produce action potentials. However, they can change their intracellular calcium concentration and produce calcium waves (Verkhratsky et al., 2012). This process (often termed "calcium excitability") triggers gliotransmitter release. The best characterized gliotransmitters are glutamate, adenosine triphosphate (ATP), adenosine, $\gamma$ amino butyric acid (GABA), D-serine, and taurine. Astrocytes also release interleukin-1 (IL-1), tumor necrosis factor $\alpha$ $(\mathrm{TNF} \alpha)$, neurotrophins, and prostaglandins (see Frank, 2013). Gliotransmitters are released by several mechanisms: either via calcium-dependent exocytosis (Parpura et al., 1994; Bezzi et al., 2004; Martineau, 2013), or transporters and channels such as cystine-glutamate antiport (Warr et al., 1999), connexon/pannexon hemichannels (Cotrina et al., 1998; Ye et al., 2003), ionotropic purinergic receptors (Duan et al., 2003), reverse mode of glutamate-, and GABA-transporters (Szatkowski et al., 1990; Gallo et al., 1991), volume-regulated anion channels and organic anion transporters (Rosenberg et al., 1994; Wang et al., 2002).

Astrocytes have complex functions in the central nervous system (CNS), including maintenance of the extracellular milieu for optimal neuronal function, regulation of synaptic plasticity, and participation in neuromodulatory actions. They contribute to sleep homeostasis, synchronization of neuronal networks, cortical oscillatory activity, chemosensitivity, and regulation of brain metabolism (Gourine et al., 2010; Halassa and Haydon, 2010; Henneberger et al., 2010; Poskanzer and Yuste, 2011; Navarrete et al., 2012; Frank, 2013; Lee et al., 2014). Astrocytes are affected in several pathophysiological processes such as neurodegenerative disorders, stroke, epilepsy, Alzheimer's, Huntington's and Parkinson's disease, amyotrophic lateral sclerosis, frontotemporal dementia, anxiety or primary cerebellar atrophy (Tian et al., 2005; Halassa and Haydon, 2010; Han et al., 2012; Shan et al., 2012; Orr et al., 2015; Sica, 2015; Zimmer et al., 2015).

As several aspects of astrocytic functions were thoroughly covered by numerous reviews, in this mini-review, I would like to focus on a distinct form of astrocyte-neuron communication, namely astrocytic actions on neuronal extrasynaptic currents.

\section{NEURONAL EXTRASYNAPTIC CURRENTS AND THEIR ASTROCYTIC CONTROL}

Extrasynaptic currents can be detected in several areas of the CNS and show large heterogeneity in their kinetics, direction, neuro/gliotransmitters, and receptors responsible for them. These currents are elicited by ambient neurotransmitters acting on extrasynaptic neuro/gliotransmitter receptors. Ambient neurotransmitter levels are under control of both neurons and astrocytes, and have several potential sources such as spillover from synaptic clefts, neuronal volume transmission or somatodendritic release by neurons, and uptake or release of neuro/gliotransmitters by astrocytes (e.g., Semyanov et al., 2004; Okubo and Iino, 2011).

The following sections will focus on the astrocytic influences on extrasynaptic currents which will be grouped according to their time scale and direction.

\section{Slow Inward Currents: The "Phasic" Extrasynaptic Action}

Neuronal slow inward currents (SICs) are phasic extrasynaptic excitatory events distinguished from excitatory postsynaptic currents (EPSCs), due to differences in amplitude, rise time and decay time. The amplitude of these currents was 18-477 pA, with slow rise (13-332 ms) and decay times ( $72-1630 \mathrm{~ms})$, fit by a single exponential function. In contrast, the amplitude of the miniature EPSCs was 19-40 pA, the rise time was significantly shorter (1-6 ms), whereas decay had a double exponential fit $\left(\tau_{1}=6.6-27.6 \mathrm{~ms}, \tau_{2}=83-146 \mathrm{~ms}\right.$; Fellin et al., 2004; Shigetomi et al., 2008; Bardoni et al., 2010; Reyes-Haro et al., 2010).

These currents are generated by activation of extrasynaptic NMDA receptors containing NR2B subunits, as SICs were prevented by general or NR2B subunit selective NMDA receptor antagonists. The involvement of NMDA receptors is also supported by the observation that appearance of SICs is largely facilitated in $\mathrm{Mg}^{2+}$-free extracellular solution. D-serine, a coactivator of the NMDA receptor also contributed to generation of SICs (Angulo et al., 2004; Fellin et al., 2004; Kozlov et al., 2006; D’Ascenso et al., 2007; Nie et al., 2010; Reyes-Haro et al., 2010; Pirttimaki et al., 2011; Pirttimaki and Parri, 2012; Figure 1).

SICs were found in several areas of the CNS, such as in the hippocampus (Angulo et al., 2004; Fellin et al., 2004; Perea and Araque, 2005; Carmignoto and Fellin, 2006), visual cortex (Chen et al., 2012; Perea et al., 2014), olfactory bulb (Kozlov et al., 2006), nucleus accumbens (D’Ascenso et al., 2007), thalamus (Parri et al., 2001), medial nucleus of the trapezoid body (ReyesHaro et al., 2010), or the dorsal horn of the spinal cord (Bardoni et al., 2010; Nie et al., 2010).

It has been extensively demonstrated that SICs are consequences of astrocytic activity. Stimulation of astrocytes in astrocyte-neuron co-cultures led to the appearance of SICs on neurons in their neighborhood, and inhibition of astrocytic calcium signaling prevented the development of these events (Araque et al., 1998). When astrocytic intracellular calcium concentration was increased in slice preparations by $\mathrm{Ca}^{2+}$ uncaging, group I and II metabotropic glutamate receptor (mGluR) or muscarinic acetylcholine receptor agonists, ATP, prostaglandin $\mathrm{E}_{2}\left(\mathrm{PGE}_{2}\right)$, or optogenetic activation of astrocytes, the frequency of neuronal SICs was significantly increased (Angulo et al., 2004; Fellin et al., 2004; Perea and Araque, 2005; D’Ascenso et al., 2007; Bardoni et al., 2010; Pirttimaki et al., 2011; Chen et al., 2012; Perea et al., 2014). However, not all effects increasing astrocytic intracellular calcium concentration generated SICs on neurons: both $\mathrm{P}_{2} \mathrm{Y}_{1}$ and PAR-1 receptor stimulation on hippocampal astrocytes led to elevation of intracellular $\mathrm{Ca}^{2+}$, but only the latter one elicited SICs on pyramidal cells (Shigetomi et al., 2008). Blockade of action 


\begin{tabular}{|c|c|}
\hline \multicolumn{2}{|c|}{ Figure legends } \\
\hline 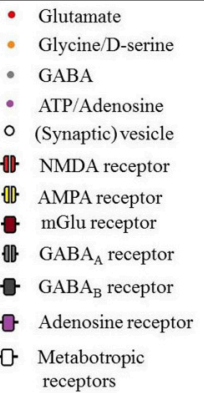 & 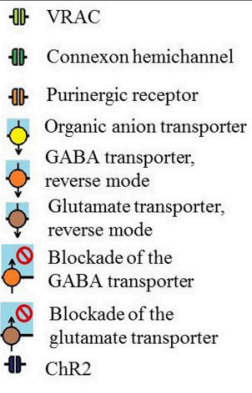 \\
\hline
\end{tabular}

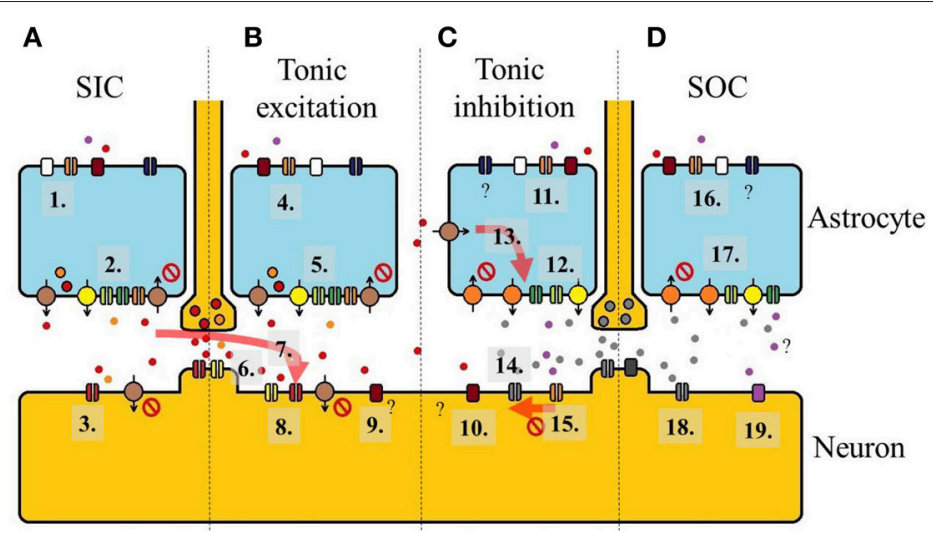

FIGURE 1 | Summary of the astrocytic actions on neuronal extrasynaptic currents. (A) Slow inward current (SIC). (1) Astrocytes can be activated by purinoreceptors (e.g., P2 $\mathrm{X}_{7}$ ), metabotropic glutamate receptors (mGluRs), other metabotropic receptors or channelrhodopsin-2 (ChR2) expressed in an astrocyte-specific way. (2) Glutamate (and D-serine) is released by astrocytes. Besides exocytosis of vesicles containing gliotransmitters, the reverse mode of glutamate transporters EAAT-1 and -2, organic anion transporters, volume-regulated anion channels (VRAC), connexon hemichannels, ionotropic purinergic receptors, or blockade of glutamate transporters are the molecules responsible for glutamate release. (3) Glutamate (and D-serine) diffuses to a neuron close to the release site and binds to extrasynaptic NMDA receptors. The activation of these receptors leads to SICs. Note that blockade of neuronal EAAT-2 can also contribute to the elevation of ambient glutamate levels eliciting SICs. (B) Tonic excitatory current. (4) Astrocytic activation via purinoreceptors (e.g., P2X 7 ), mGluRs, other metabotropic receptors or channelrhodopsin-2 (see 1). (5) Release of glutamate and co-activators like D-serine (see 2). (6) Glutamate spillover from synaptic clefts (and volume transmission) also increases extrasynaptic glutamate concentration. (7) Diffusion of glutamate leading to SICs in a location closer to its release site can elicit tonic inward current. (8) Activation of extrasynaptic NMDA receptors, AMPA receptors and extrasynaptic mGluRs (9) lead to tonic excitatory currents. (C) Tonic inhibitory current. (10) Glutamate acting on a different set of mGluRs can elicit outward currents. (11) Activation of astrocytes by different receptors. Although astrocytes activated by ChR2 can potentially generate outward currents on certain neurons, it has not been directly shown. (12) GABA is released by GABA transporters in reverse mode (or by inhibition of the normal mode), VRACs or organic anion transporters. ATP is released via connexon hemichannels. (13) Increase of ambient glutamate concentration facilitates glutamate uptake and GABA release. (14) GABA released by astrocytes or resulted from volume transmission or spillover from

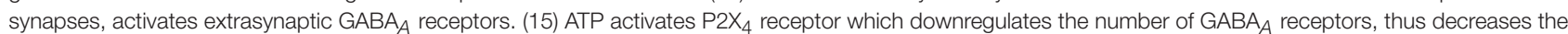
amplitude of tonic outward current. (D) Slow outward currents (SOCs). (16) Astrocytic activation (see 11). (17) Release of GABA and ATP/adenosine (see 12). (18) SOCs are generated by activation of extrasynaptic GABAA receptors. (19) SOC-like hyperpolarizing events can be seen by activation of A1 adenosine receptor. Adenosine stimulating these receptors likely has non-neuronal origin. A scheme of an excitatory synapse can be seen between panels $\mathbf{A}$ and $\mathbf{B}$, whereas an inhibitory synapse is shown between panels $\mathbf{C}$ and $\mathbf{D}$

potential firing or release of synaptic vesicles did not affect the amplitude and frequency of SICs (Araque et al., 1998; Angulo et al., 2004; Fellin et al., 2004; Perea and Araque, 2005; D’Ascenso et al., 2007; Pirttimaki et al., 2011).

SICs recorded from different areas of the CNS have largely variable characteristics (Table 1) The large variance in their kinetic parameters implicate that gliotransmitters eliciting these events originate from nonsynaptic sources in a variable distance from their targets (Carmignoto and Fellin, 2006), and gliotransmitter concentration and the number of involved receptors shape the kinetics of SICs. Elevation of ambient glutamate concentration by the glutamate transporter inhibitor TBOA increased the amplitude of SICs (Angulo et al., 2004), and their rise and decay times became slower (Fellin et al., 2004).

The functional role of SICs is likely the synchronization of neighboring neurons after longer excitatory stimulation by a significant input of their area. Longer stimulation of inputs increased the frequency of SICs in the nucleus accumbens, the hippocampus, and the ventrobasal thalamus. This synchronization was mostly demonstrated in forebrain structures: astrocytes can elicit SICs synchronously on pairs of neurons in the nucleus accumbens and on the hippocampal CA1 pyramidal neurons (Angulo et al., 2004; Fellin et al., 2004; D’Ascenso et al., 2007; Pirttimaki et al., 2011). In contrast, synchronization of SICs occurred rarely in the brainstem (ReyesHaro et al., 2010).

SICs can be affected by a special form of plasticity: prolonged stimulation of afferents of the ventrobasal thalamus resulted a longer lasting (at least $60 \mathrm{~min}$ long) increase of SIC frequency, representing an astrocyte-dependent nonsynaptic plasticity (Pirttimaki et al., 2011).

\section{Slow Outward Currents: "Phasic" Inhibition by Astrocytes}

Slow outward currents (SOCs) are rarely described phasic inhibitory events. Distinguishing them from neuronal inhibitory postsynaptic currents (IPSCs) is not as clear as in the case of SICs: although with their rise time of 26-109 ms and decay time of $350 \mathrm{~ms}$ they are significantly slower than $\mathrm{GABA}_{\mathrm{A}}$ receptormediated fast IPSCs (3-8 ms decay time), their kinetic properties are closer to slow IPSCs mediated by $\mathrm{GABA}_{\mathrm{A}}(30-70 \mathrm{~ms}$ decay time; Capogna and Pearce, 2011) and $\mathrm{GABA}_{\mathrm{B}}$ receptors $(80-$ $100 \mathrm{~ms}$ rise time, $180-428 \mathrm{~ms}$ decay time; Degro et al., 2015).

GABA acting on ( $\delta$-subunit-containing) $\mathrm{GABA}_{\mathrm{A}}$ receptors is responsible for generation of SOCs (Kozlov et al., 2006; JiménezGonzález et al., 2011; Le Meur et al., 2012; Pirttimaki et al., 2013). 


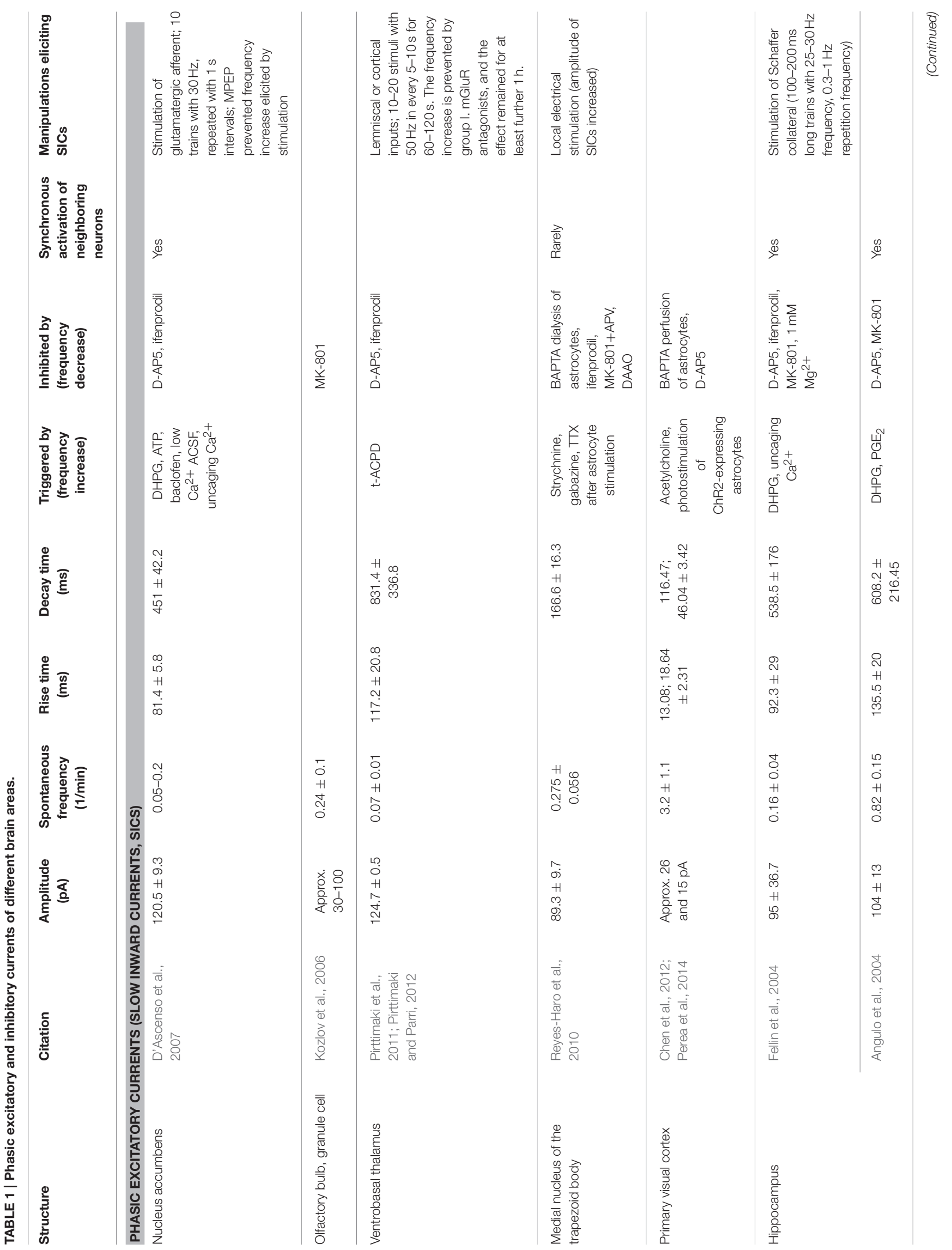




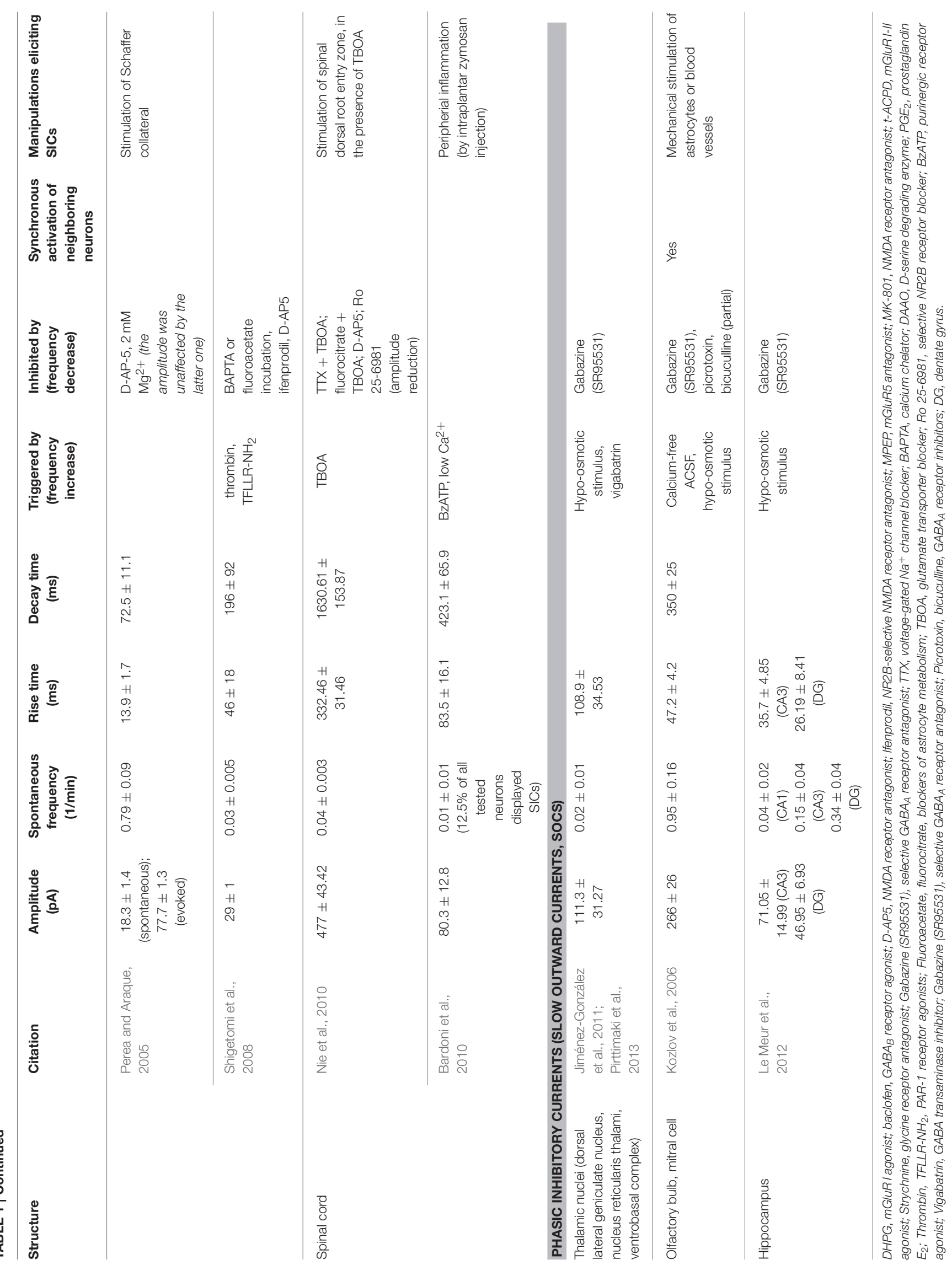


SOCs were recorded from the ventrobasal thalamus (JiménezGonzález et al., 2011), the olfactory bulb (Kozlov et al., 2006), and from the hippocampus (Le Meur et al., 2012). SOCs can appear on the same neurons where SICs were also recorded: spontaneous inhibitory events had a significantly lower frequency than SICs in the ventrobasal thalamus and the hippocampal CA1 region, but SICs and SOCs had similar frequencies in the CA3 region and in the dentate gyrus (Jiménez-González et al., 2011; Le Meur et al., 2012).

The astrocytic origin of SOCs was demonstrated in the olfactory bulb, where mechanical stimulation of astrocytes or blood vessels was capable of eliciting these events (Kozlov et al., 2006). Contribution of astrocytes to SOCs was also shown by pharmacological blockade of neuronal activity and vesicle release, or facilitation of gliotransmitter release (Kozlov et al., 2006; Jiménez-González et al., 2011; Le Meur et al., 2012; Table 1; Figure 1).

Although not discussed as SOCs, astrocytic activity might raise other inhibitory events with slow kinetics. Rhythmical, hyperpolarizing, $A_{1}$ adenosine-receptor mediated events were found in thalamic nuclei of the cat. The possible source of adenosine was the ATP released by non-neuronal structures (Lörincz et al., 2009).

The significance of the rarely identified SOCs is not known in great detail. However, they might have a role in neuronal synchronization, as they appeared synchronously on neurons of the olfactory bulb (Kozlov et al., 2006) and displayed reduced amplitude and slower kinetics in thalamocortical neurons from absence seizure model rats (Pirttimaki et al., 2013).

\section{Tonic Excitatory Currents Regulated by Astrocytes}

Neuronal tonic excitatory currents were detected in several brain structures, but the relationship between these currents and astrocytic activity is less clear than with SICs. These currents are predominantly mediated by ambient glutamate and the NMDA receptor co-activator glycine (Le Meur et al., 2007; Papouin et al., 2012). Glutamate can originate both from neurotransmitter spillover and astrocytic release. Furthermore, ambient glutamate concentration can be decreased by uptake via different classes of glutamate transporters (Excitatory Amino Acid Transporters; EAAT1-4). EAAT1 and EAAT2 are predominantly glial, whereas EAAT3 and EAAT4 are neuronal (see Okubo and Iino, 2011; Zhou and Danbolt, 2013). Neuronal receptors of ambient glutamate are also heterogeneous: although extrasynaptic NMDA receptors contribute to the majority of tonic excitatory currents (Jabaudon et al., 1999; Angulo et al., 2004; Fellin et al., 2006; Le Meur et al., 2007; Fleming et al., 2011; Papouin et al., 2012; Petralia, 2012; Papouin and Oliet, 2014), involvement of AMPA receptors (Sasaki et al., 2012; Beppu et al., 2014) or group II mGluRs (Kõszeghy et al., 2015) was also demonstrated.

Tonic excitatory currents might appear together with SICs. The glutamate transporter inhibitor TBOA increased the amplitude of SICs together with activation of an NMDA-receptor dependent tonic current. It is likely that glutamate originating from the same astrocytic source elicited SICs on its closer targets, but activated a larger number of NMDA receptors with a lower concentration on distant activation sites on neurons, thus eliciting tonic inward current (Jabaudon et al., 1999; Angulo et al., 2004).

Tonic excitatory currents were detected in the hippocampus (Jabaudon et al., 1999; Angulo et al., 2004; Fellin et al., 2006; Le Meur et al., 2007; Papouin et al., 2012), the supraoptic nucleus (Fleming et al., 2011), the dorsal horn of the spinal cord (Nie et al., 2010), the pedunculopontine nucleus (Kõszeghy et al., 2015), and on cerebellar Purkinje cells (Sasaki et al., 2012).

Astrocytic contribution to tonic inward currents is supported by several indirect and direct observations. Inhibition of astrocytic functions by gliotoxins, EAAT1 and 2 glutamate transporters or blockade of astrocytic glutamine synthase increased the amplitude of the tonic inward current (Jabaudon et al., 1999; Angulo et al., 2004; Le Meur et al., 2007; Fleming et al., 2011). Stimulation of astrocytic $\mathrm{P} 2 \mathrm{X}_{7}$ receptors or gliotransmitter release resulted the same effect (Fellin et al., 2004). As direct evidence for glial contribution, optogenetic stimulation of Bergmann glia elicited tonic inward neuronal current (Sasaki et al., 2012; Beppu et al., 2014). Optogenetic stimulation of astrocytes caused tonic depolarization and increased firing rate on neurons of the retrotrapezoid nucleus (Figueiredo et al., 2011) and the visual cortex (Perea et al., 2014). In these latter cases, the presence of tonic inward current was not demonstrated, but this current likely contributed to the observed phenomenon (Table 2, Figure 1).

With their regulatory role on tonic neuronal excitatory currents, astrocytes participate in protection of neurons against hyperexcitability, are involved in the pathogenesis of seizures, contribute to long term synaptic plasticity and learning, and regulate neuromodulatory actions.

Administration of the glutamate transporter inhibitor TBOA generated seizures (Montiel et al., 2005), and selective astrocytic activation and consequential glutamate release triggered epileptiform discharges (Kang et al., 2005). Stimulation of astrocytic cannabinoid type 1 (CB1) receptors induced prolonged epileptic activity (Coiret et al., 2012), and conditional deletion of EAAT2 in astrocytes led to seizures (Petr et al., 2015).

In cooperation with neuronal processes, astrocytic regulation of these extrasynaptic currents contributes to learning and synaptic plasticity. Mice heterozygous for the EAAT2 gene (with moderate loss of EAAT2 protein) exhibited altered learning abilities compared to wild type animals (improvement in cue-based fear conditioning, but worse context-based fear conditioning; Kiryk et al., 2008). Long-term depression (but not long term potentiation) needed activation of both synaptic and extrasynaptic NMDA receptors (by glutamate and its co-agonists, glycine and D-serine; Papouin et al., 2012), demonstrating the role of neuronal and astrocytic cooperation in synaptic plasticity.

Tonic excitatory currents elicited with the contribution of astrocytic activity are neuromodulatory mechanisms. Tonic excitatory currents appeared after stimulation of astrocytic muscarinic acetylcholine receptors and induced acetylcholinedependent cortical plasticity (Chen et al., 2012). Astrocyteand mGluR-dependent tonic inward and outward currents in endocannabinoid signaling were also demonstrated in the pedunculopontine nucleus (Kõszeghy et al., 2015). 


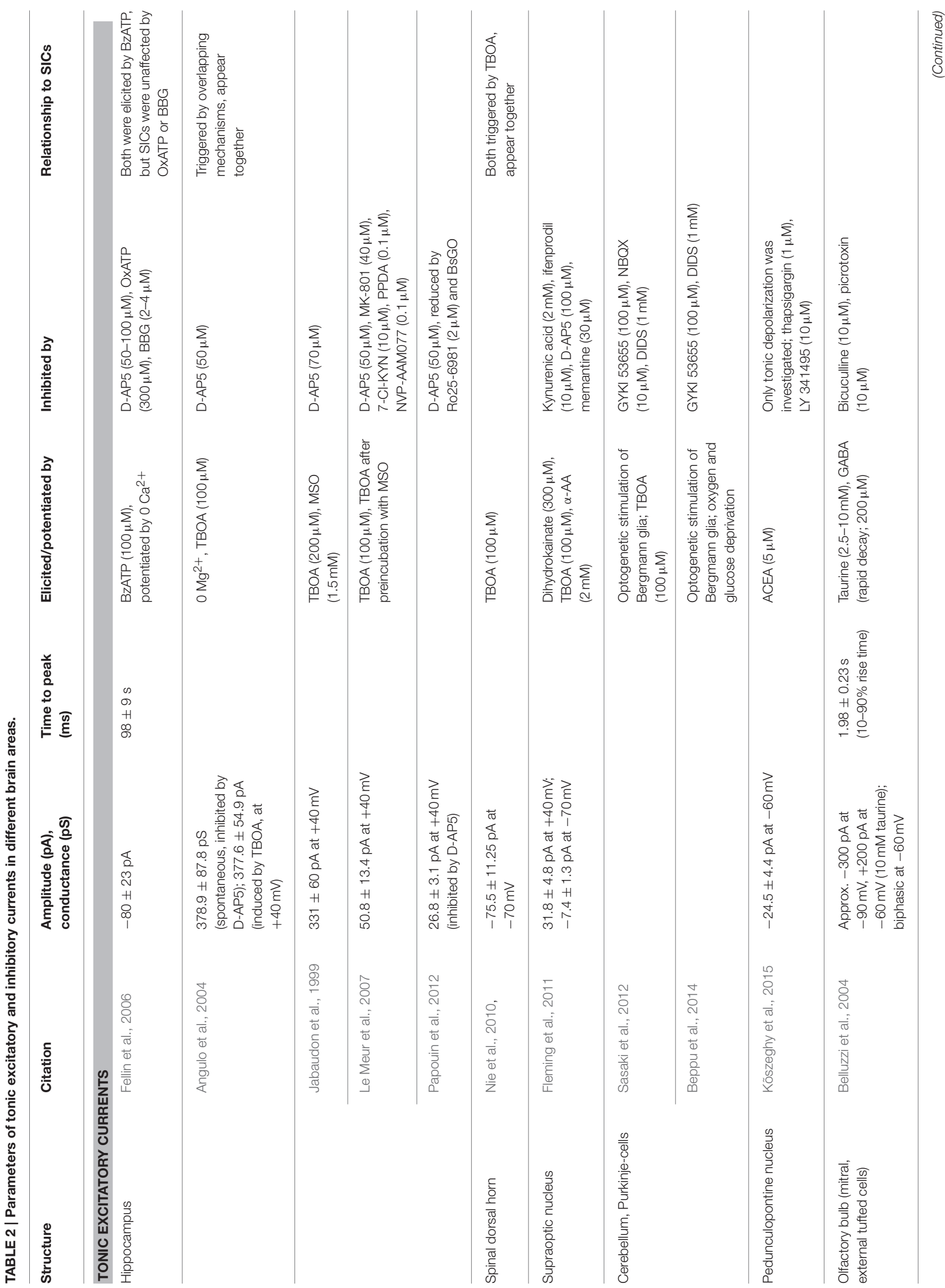




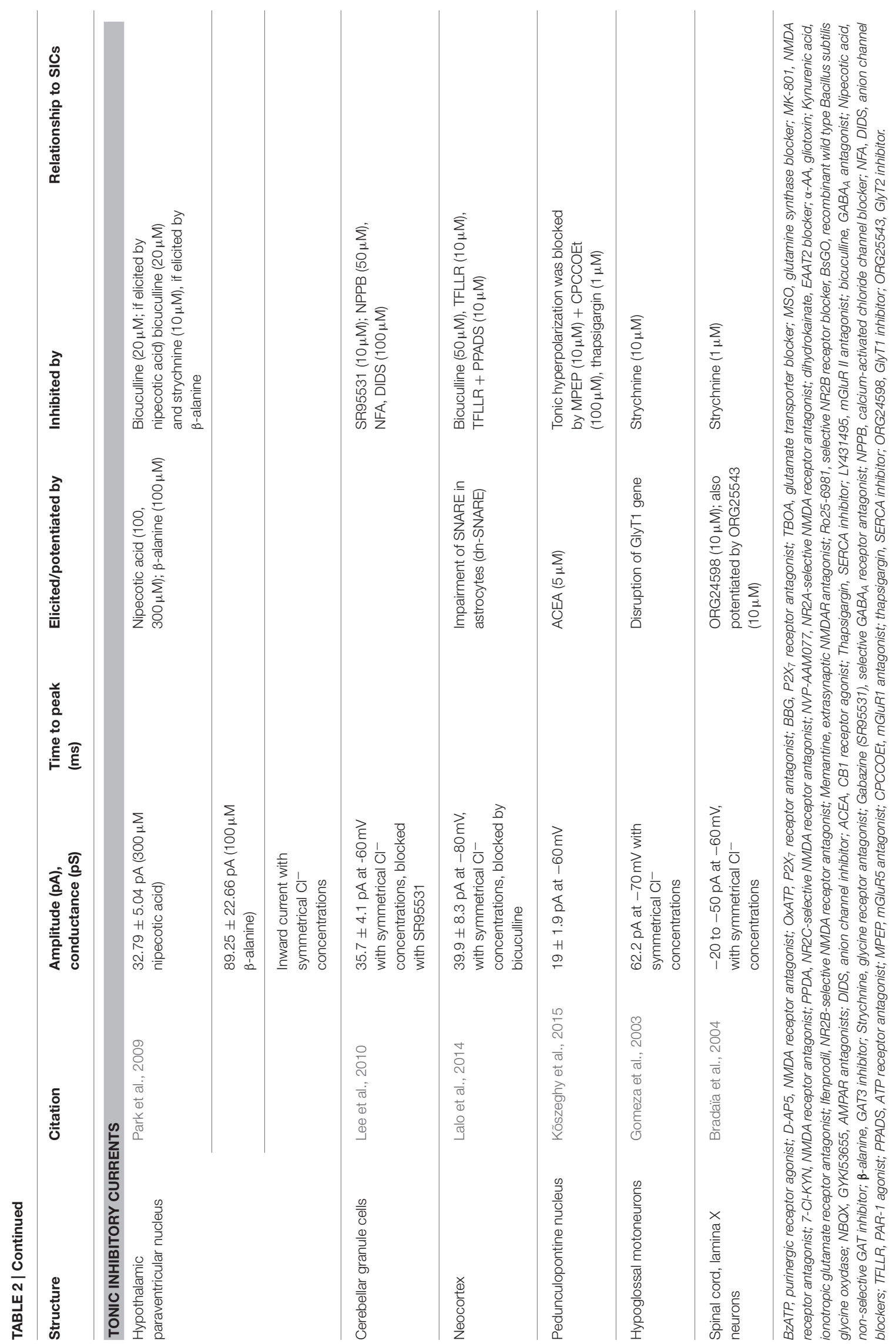




\section{Tonic Inhibitory Currents by Astrocytes}

Neuronal tonic inhibitory currents mainly originate from activation of extrasynaptic $\mathrm{GABA}_{\mathrm{A}}$ receptors (reviewed in: Semyanov et al., 2004; Cellot and Cherubini, 2013; Connelly et al., 2013; Lee and Maguire, 2014). Briefly, tonic GABAmediated currents are present in several brain areas (cortex, hippocampus, cerebellum, thalamus, striatum, and brainstem). The structure of receptors responsible for tonic $\mathrm{GABA}_{\mathrm{A}}$ currents might differ from the ones responsible for IPSCs. Extrasynaptic receptors consist mostly of $\delta$-subunits, but $\gamma$ subunits can also contribute to the receptor composition in certain cases.

Similar to tonic inward currents, tonic GABAergic currents are not exclusively of glial origin. Ambient GABA can originate from neurotransmitter spillover or volume transmission as well, although astrocytes can also release GABA as a gliotransmitter via different mechanisms (through bestrophin channels, Lee et al., 2010; reverse mode of GABA transporters GAT2 and GAT3, Angulo et al., 2008). Glutamate uptake by astrocytes was also coupled with GABA release (Héja et al., 2012).

Tonic GABA currents can be regulated by astrocytes via ATP release. ATP promoted the down-regulation of $\mathrm{GABA}_{\mathrm{A}}$ receptors via activation of the $\mathrm{P}_{2} \mathrm{X}_{4}$ receptor, and thus attenuated the effects of extrasynaptic GABA in the neocortex (Lalo et al., 2014).

In contrast to astrocyte-dependent tonic excitation, it has not been shown that optogenetic activation of astrocytes elicits hyperpolarization of neurons. However, based on in vivo measurements of the firing rates, certain neurons of the visual cortex responded with decrease of their firing rate to optogenetic stimulation of astrocytes (Perea et al., 2014). Although this change of activity can be the consequence of actions on network activity, the finding is at least not against the possibility that astrocytic activation can cause neuronal hyperpolarization.

Tonic inhibitory currents can be elicited by neuro/gliotransmitters other than GABA. Tonic glycinergic currents were generated on spinal cord neurons by either blockade of glial glycine transporter (Bradaïa et al., 2004) or on hypoglossal motoneurons by disruption of the GLYT1 gene (Gomeza et al., 2003). Tonic hyperpolarization and tonic outward currents activated by CB1 receptor agonists on certain neurons of the pedunculopontine nucleus were successfully prevented by blockers of group I mGluRs (Kõszeghy et al., 2015; Table 2; Figure 1).

The tonic outward current has variable roles in regulation of neuronal functions, such as setting neuronal excitability, contribution to network oscillations, and has developmental functions like inhibition of cell proliferation and stimulation of cell migration (Semyanov et al., 2004; Park et al., 2009; Connelly et al., 2013; Lee and Maguire, 2014).

\section{REFERENCES}

Angulo, M. C., Kozlov, A. S., Charpak, S., and Audinat, E. (2004). Glutamate released from glial cells synchronizes neuronal activity in the hippocampus. J. Neurosci. 24, 6920-6927. doi: 10.1523/JNEUROSCI.047 3-04.2004

\section{CONCLUDING REMARKS}

Astrocyte-dependent neuronal extrasynaptic currents seem to be a general feature of the CNS. These phenomena are not always consequences of astrocytic actions on neurons, but often represent interplay between neuronal and astrocytic activity. The degree of astrocytic contribution is different: SICs have unambiguous astrocytic origin, but the astrocytic component of tonic inward and outward currents can't be always clearly separated.

The (patho)physiological roles of these currents are also heterogeneous and sometimes contradictory: tonic inward and outward currents are generally thought to contribute to synaptic plasticity and memory, although similar neuronal tonic inward currents can be observed in models of Alzheimer's disease (Talantova et al., 2013). Astrocytic functions can be responsible for neuroprotective mechanisms via regulation of neuronal excitability; but, under pathological conditions, such as stroke or epilepsy, astrocytic malfunction aggravates neuronal injury by causing neurotoxicity (Tian et al., 2005; Seifert and Steinhäuser, 2013; Beppu et al., 2014). Astrocyte-dependent tonic currents are also parts of neuromodulatory mechanisms, where both excitation and inhibition can be seen on neurons from the same neurochemical subgroup (Kõszeghy et al., 2015).

Furthermore, the function of the generally observed SICs is also not fully resolved. Appearing on neighboring neurons at the same time and being elicited by well-defined afferentation of the investigated brain area, they can synchronize neuronal activity, but their role in synaptic plasticity, to the best of my knowledge, has not been thoroughly investigated yet.

Taken together, neuronal extrasynaptic currents influenced by astrocytes contribute to several pathophysiological processes, but the uniform role of these phenomena and the significance of pure astrocytic mechanisms in eliciting them still remain an exciting field for further investigations.

\section{AUTHOR CONTRIBUTIONS}

BP wrote the paper.

\section{ACKNOWLEDGMENTS}

This work was supported by the János Bolyai Research Scholarship of the Hungarian Academy of Sciences, the Szodoray Fellowship of the University of Debrecen and the National Brain Research Program (KTIA_13_NAP-A-I/10.). I am indebted to Dr. Attila Szöllõsi (University College Dublin, Ireland) for the thorough reading of the manuscript and for his valuable comments. 
Araque, A., Parpura, V., Sanzgiri, R. P., and Haydon, P. G. (1998). Glutamatedependent astrocyte modulation of synaptic transmission between cultured hippocampal neurons. Eur. J. Neurosci. 10, 2129-2142. doi: 10.1046/j.14609568.1998.00221. $\mathrm{x}$

Araque, A., Parpura, V., Sanzgiri, R. P., and Haydon, P. G. (1999). Tripartite synapses: glia, the unacknowledged partner. Trends Neurosci. 22, 208-215. doi: 10.1016/S0166-2236(98)01349-6

Bardoni, R., Ghirri, A., Zonta, M., Betelli, C., Vitale, G., Ruggieri, V., et al. (2010). Glutamate-mediated astrocyte-to-neuron signalling in the rat dorsal horn. J. Physiol. 588(Pt 5), 831-846. doi: 10.1113/jphysiol.2009.180570

Belluzzi, O., Puopolo, M., Benedusi, M., and Kratskin, I. (2004). Selective neuroinhibitory effects of taurine in slices of rat main olfactory bulb. Neuroscience 124, 929-944. doi: 10.1016/j.neuroscience.2003.12.032

Beppu, K., Sasaki, T., Tanaka, K. F., Yamanaka, A., Fukazawa, Y., Shigemoto, R., et al. (2014). Optogenetic countering of glial acidosis suppresses glial glutamate release and ischemic brain damage. Neuron 81, 314-320. doi: 10.1016/j.neuron.2013.11.011

Bezzi, P., Gundersen, V., Galbete, J. L., Seifert, G., Steinhäuser, C., Pilati, E., et al. (2004). Astrocytes contain a vesicular compartment that is competent for regulated exocytosis of glutamate. Nat. Neurosci. 7, 613-620. doi: $10.1038 / \mathrm{nn} 1246$

Bradaïa, A., Schlichter, R., and Trouslard, J. (2004). Role of glial and neuronal glycine transporters in the control of glycinergic and glutamatergic synaptic transmission in lamina X of the rat spinal cord. J. Physiol. 559(Pt 1), 169-186. Erratum in: J. Physiol. 559(Pt 3), 985. doi: 10.1113/jphysiol.2004.068858

Capogna, M., and Pearce, R. A. (2011). GABA $A_{\text {,slow }}$ : causes and consequences. Trends Neurosci. 34, 101-112. doi: 10.1016/j.tins.2010.10.005

Carmignoto, G., and Fellin, T. (2006). Glutamate release from astrocytes as a non-synaptic mechanism for a neuronal synchronization in the hippocampus. J. Physiol. (Paris) 99, 98-102. doi: 10.1016/j.jphysparis.2005.12.008

Cellot, G., and Cherubini, E. (2013). Functional role of ambient GABA in refining neuronal circuits early in postnatal development. Front. Neural Circuits 7:136. doi: 10.3389/fncir.2013.00136

Chen, N., Sugihara, H., Sharma, J., Perea, G., Petravicz, J., Le, C., et al. (2012). Nucleus basalis-enabled stimulus-specific plasticity in the visual cortex is mediated by astrocytes. Proc. Natl. Acad. Sci. U.S.A. 109, E2832-E2841. doi: 10.1073/pnas.1206557109

Coiret, G., Ster, J., Grewe, B., Wendling, F., Helmchen, F., Gerber, U., et al. (2012). Neuron to astrocyte communication via cannabinoid receptors is necessary for sustained epileptiform activity in rat hippocampus. PLOS ONE 7:e37320. doi: 10.1371/journal.pone.0037320

Connelly, W. M., Errington, A. C., Di Giovanni, G., and Crunelli, V. (2013). Metabotropic regulation of extrasynaptic $\mathrm{GABA}_{A}$ receptors. Front. Neural Circuits 7:171. doi: 10.3389/fncir.2013.00171

Cotrina, M. L., Lin, J. H., Alves-Rodrigues, A., Liu, S., Li, J., Azmi-Ghadimi, H., et al. (1998). Connexins regulate calcium signaling by controlling ATP release. Proc. Natl. Acad. Sci. U.S.A. 95, 15735-15740. doi: 10.1073/pnas.95.26.15735

D’Ascenso, M., Fellin, T., Terunuma, M., Revilla-Sancez, R., Meaney, D. F., Aubertson, Y. P., et al. (2007). mGluR5 stimulates gliotransmission in the nucleus accumbens. Proc. Natl. Acad. Sci. U.S.A. 104, 1995-2000. doi: 10.1073/pnas.0609408104

Degro, C. E., Kulik, A., Booker, S. A., and Vida, I. (2015). Compartmental distribution of GABAB receptor-mediated currents along the somatodendritic axis of hippocampal principal cells. Front. Synaptic Neurosci. 7:6. doi: 10.3389/fnsyn.2015.00006

Duan, S., Anderson, C. M., Keung, E. C., Chen, Y., Chen, Y., and Swanson, R. A. (2003). P2X7 receptor-mediated release of excitatory amino acids from astrocytes. J. Neurosci. 23, 1320-1328.

Fellin, T., Pascual, O., Gobbo, S., Pozzan, T., Haydon, P. G., and Carmignoto, G. (2004). Neuronal synchrony mediated by astrocytic glutamate through activation of extrasynaptic NMDA receptors. Neuron 43, 729-743. doi: 10.1016/j.neuron.2004.08.011

Fellin, T., Pozzan, T., and Carmignoto, G. (2006). Purinergic receptors mediate two distinct glutamate release pathways in hippocampal astrocytes. J. Biol. Chem. 281, 4274-4284. doi: 10.1074/jbc.M510679200

Figueiredo, M., Lane, S., Tang, F., Liu, B. H., Hewinson, J., Marina, N., et al. (2011). Optogenetic experimentation on astrocytes. Exp. Physiol. 96, 40-50. doi: 10.1113/expphysiol.2010.052597
Fleming, T. M., Scott, V., Naskar, K., Joe, N., Brown, C. H., and Stern, J. E. (2011) State-dependent changes in astrocyte regulation of extrasynaptic NMDA receptor signalling in neurosecretory neurons. J. Physiol. 589, 3929-3941. doi: 10.1113/jphysiol.2011.207340

Frank, M. G. (2013). Astroglial regulation of sleep homeostasis. Curr. Opin. Neurobiol. 23, 812-818. doi: 10.1016/j.conb.2013.02.009

Gallo, V., Patrizio, M., and Levi, G. (1991). GABA release triggered by the activation of neuron-like non-NMDA receptors in cultured type 2 astrocytes is carrier-mediated. Glia 4, 245-255. doi: 10.1002/glia.440 040302

Gomeza, J., Hülsmann, S., Ohno, K., Eulenburg, V., Szöke, K., Richter, D., et al. (2003). Inactivation of the glycine transporter 1 gene discloses vital role of glial glycine uptake in glycinergic inhibition. Neuron 40, 785-796. Erratum in: Neuron 41, 675. doi: 10.1016/S0896-6273(03)00672-X

Gourine, A. V., Kasymov, V., Marina, N., Tang, F., Figueiredo, M. F., Lane, S., et al. (2010). Astrocytes control breathing through pH-dependent release of ATP. Science 329, 571-575. doi: 10.1126/science.1190721

Halassa, M. M., and Haydon, P. G. (2010). Integrated brain circuits: astrocytic networks modulate neuronal activity and behaviour. Annu. Rev. Physiol. 72, 335-355. doi: 10.1146/annurev-physiol-021909-135843

Han, J., Kesner, P., Metna-Laurent, M., Duan, T., Xu, L., Georges, F., et al. (2012). Acute cannabinoids impair working memory through astroglial CB1 receptor modulation of hippocampal LTD. Cell 148, 1039-1050. doi: 10.1016/j.cell.2012.01.037

Héja, L., Nyitrai, G., Kékesi, O., Dobolyi, A., Szabó, P., Fiáth, R., et al. (2012). Astrocytes convert network excitation to tonic inhibition of neurons. BMC Biol. 10:26. doi: 10.1186/1741-7007-10-26

Henneberger, C., Papouin, T., Oliet, S. H., and Rusakov, D. A. (2010). Longterm potentiation depends on release of D-serine from astrocytes. Nature 463, 232-236. doi: 10.1038/nature08673

Jabaudon, D., Shimamoto, K., Yasuda-Kamatani, Y., Scanziani, M., Gähwiler, B. H., and Gerber, U. (1999). Inhibition of uptake unmasks rapid extracellular turnover of glutamate of nonvesicular origin. Proc. Natl. Acad. Sci. U.S.A. 96, 8733-8738. doi: 10.1073/pnas.96.15.8733

Jiménez-González, C., Pirttimaki, T., Cope, D. W., and Parri, H. R. (2011). Non-neuronal, slow GABA signalling in the ventrobasal thalamus targets $\delta$ subunit-containing $\mathrm{GABA}_{A}$ receptors. Eur. J. Neurosci. 33, 1471-1482. doi: 10.1111/j.1460-9568.2011.07645.x

Kang, N., Xu, J., Xu, Q., Nedergaard, M., and Kang, J. (2005). Astrocytic glutamate release-induced transient depolarization and epileptiform discharges in hippocampal CA1 pyramidal neurons. J. Neurophysiol. 94, 4121-4130. doi: 10.1152/jn.00448.2005

Kiryk, A., Aida, T., Tanaka, K., Banerjee, P., Wilczynski, G. M., Meyza, K., et al. (2008). Behavioral characterization of GLT1 $(+/-)$ mice as a model of mild glutamatergic hyperfunction. Neurotox. Res. 13, 19-30. doi: 10.1007/BF03033364

Kõszeghy, Á., Kovács, A., Bíró, T., Szücs, P., Vincze, J., Hegyi, Z., et al. (2015). Endocannabinoid signaling modulates neurons of the pedunculopontine nucleus (PPN) via astrocytes. Brain Struct. Funct. 220, 3023-3041. doi: 10.1007/s00429-014-0842-5

Kozlov, A. S., Angulo, M. C., Audinat, E., and Charpak, S. (2006). Target cellspecific modulation of neuronal activity by astrocytes. Proc. Natl. Acad. Sci. U.S.A. 103, 10058-10063. doi: 10.1073/pnas.0603741103

Lalo, U., Palygin, O., Rasooli-Nejad, S., Andrew, J., Haydon, P. G., and Pankratov, Y. (2014). Exocytosis of ATP from astrocytes modulates phasic and tonic inhibition in the neocortex. PLoS Biol. 12:e1001747. doi: 10.1371/journal.pbio.1001747

Le Meur, K., Galante, M., Angulo, M. C., and Audinat, E. (2007). Tonic activation of NMDA receptors by ambient glutamate of non-synaptic origin in the rat hippocampus. J. Physiol. 580(Pt 2), 373-383. doi: 10.1113/jphysiol.2006. 123570

Le Meur, K., Mendizabal-Zubiaga, J., Grandes, P., and Audinat, E. (2012). GABA release by hippocampal astrocytes. Front. Comput. Neurosci. 6:59. doi: 10.3389/fncom.2012.00059

Lee, H. S., Ghetti, A., Pinto-Duarte, A., Wang, X., Dziewczapolski, G., Galimi, F., et al. (2014). Astrocytes contribute to gamma oscillations and recognition memory. Proc. Natl. Acad. Sci. U.S.A. 111, E3343-E3352. doi: $10.1073 /$ pnas.1410893111 
Lee, S., Yoon, B. E., Berglund, K., Oh, S. J., Park, H., Shin, H. S., et al. (2010). Channel-mediated tonic GABA release from glia. Science 330, 790-796. doi: $10.1126 /$ science. 1184334

Lee, V., and Maguire, J. (2014). The impact of tonic $\mathrm{GABA}_{A}$ receptor-mediated inhibition on neuronal excitability varies across brain region and cell. Front Neural Circuits 8:3. doi: 10.3389/fncir.2014.00003

Lörincz, M. L., Bao, Y., Crunelli, V., and Hughes, S. W. (2009). ATP-dependent infra-slow $(<0.1 \mathrm{~Hz})$ oscillations in thalamic networks. PLoS ONE 4:e4447. doi: 10.1371/journal.pone.0004447

Martineau, M. (2013). Gliotransmission: focus on exocytotic release of L-glutamate and D-serine from astrocytes. Biochem. Soc. Trans. 41, 1557-1561. doi: 10.1042/BST20130195

Montiel, T., Camacho, A., Estrada-Sánchez, A. M., and Massieu, L. (2005). Differential effects of the substrate inhibitor 1-trans-pyrrolidine-2,4dicarboxylate (PDC) and the non-substrate inhibitor DL-threo-betabenzyloxyaspartate (DL-TBOA) of glutamate transporters on neuronal damage and extracellular amino acid levels in rat brain in vivo. Neuroscience 133, 667-678. doi: 10.1016/j.neuroscience.2004.11.020

Navarrete, M., Perea, G., Fernandez de Sevilla, D., Gómez-Gonzalo, M., Núnez, A., Martín, E. D., et al. (2012). Astrocytes mediate in vivo cholinergic-induced synaptic plasticity. PLoS Biol. 10:e1001259. doi: 10.1371/journal.pbio.1001259

Nie, H., Zhang, H., and Weng, H. R. (2010). Bidirectional neuron-glia interactions triggered by deficiency of glutamate uptake at spinal sensory synapses. J. Neurophysiol. 104, 713-725. doi: 10.1152/jn.00282.2010

Okubo, Y., and Iino, M. (2011). Visualization of glutamate as a volume transmitter. J. Physiol. 589(Pt 3), 481-448. doi: 10.1113/jphysiol.2010.199539

Orr, A. G., Hsiao, E. C., Wang, M. M., Ho, K., Kim, D. H., Wang, X., et al. (2015). Astrocytic adenosine receptor A2A and Gs-coupled signaling regulate memory. Nat. Neurosci. 18, 423-434. doi: 10.1038/nn.3930

Papouin, T., Ladépêche, L., Ruel, J., Sacchi, S., Labasque, M., Hanini, M., et al. (2012). Synaptic and extrasynaptic NMDA receptors are gated by different endogenous coagonists. Cell 150, 633-646. doi: 10.1016/j.cell.2012.06.029

Papouin, T., and Oliet, S. H. (2014). Organization, control and function of extrasynaptic NMDA receptors. Philos. Trans. R. Soc. Lond. B Biol. Sci. 369:20130601. doi: 10.1098/rstb.2013.0601

Park, J. B., Jo, J. Y., Zheng, H., Patel, K. P., and Stern, J. E. (2009). Regulation of tonic GABA inhibitory function, presympathetic neuronal activity and sympathetic outflow from the paraventricular nucleus by astroglial GABA transporters. J. Physiol. 587, 4645-4660. doi: 10.1113/jphysiol.2009.173435

Parpura, V., Basarsky, T. A., Liu, F., Jeftinija, K., Jeftinija, S., and Haydon, P. G. (1994). Glutamate-mediated astrocyte-neuron signalling. Nature 369, 744-747. doi: $10.1038 / 369744 \mathrm{a} 0$

Parri, H. R., Gould, T. M., and Crunelli, V. (2001). Spontaneous astrocytic Ca2+ oscillations in situ drive NMDAR-mediated neuronal excitation. Nat. Neurosci. 4, 803-812. doi: 10.1038/90507

Perea, G., and Araque, A. (2005). Properties of synaptically evoked astrocyte calcium signal reveal synaptic information processing by astrocytes. J. Neurosci. 25, 2192-2203. doi: 10.1523/JNEUROSCI.3965-04.2005

Perea, G., Navarrete, M., and Araque, A. (2009). Tripartite synapses: astrocytes process and control synaptic information. Trends Neurosci. 32, 421-431. doi: 10.1016/j.tins.2009.05.001

Perea, G., Yang, A., Boyden, E. S., and Sur, M. (2014). Optogenetic astrocyte activation modulates response selectivity of the visual cortex neurons in vivo. Nat. Commun. 5:3262. doi: 10.1038/ncomms4262

Petr, G. T., Sun, Y., Frederick, N. M., Zhou, Y., Dhamne, S. C., Hameed, M. Q., et al. (2015). Conditional deletion of the glutamate transporter GLT-1 reveals that astrocytic GLT-1 protects against fatal epilepsy while neuronal GLT-1 contributes significantly to glutamate uptake into synaptosomes. J. Neurosci. 35, 5187-5201. doi: 10.1523/JNEUROSCI.4255-14.2015

Petralia, R. S. (2012). Distribution of extrasynaptic NMDA receptors on neurons. ScientificWorldJournal 2012:267120. doi: 10.1100/2012/267120

Pirttimaki, T. M., Hall, S. D., and Parri, H. R. (2011). Sustained neuronal activity generated by glial plasticity. J. Neurosci. 31, 7637-7647. doi: 10.1523/JNEUROSCI.5783-10.2011

Pirttimaki, T. M., and Parri, H. R. (2012). Glutamatergic input-output properties of thalamic astrocytes. Neuroscience 205, 18-28. doi: 10.1016/j.neuroscience.2011.12.049

Pirttimaki, T., Parri, H. R., and Crunelli, V. (2013). Astrocytic GABA transporter GAT-1 dysfunction in experimental absence seizures. J. Physiol. 591, 823-833. doi: $10.1113 /$ jphysiol.2012.242016
Poskanzer, K. E., and Yuste, R. (2011). Astrocytic regulation of cortical UP states. Proc. Natl. Acad. Sci. U.S.A. 108, 18453-18458. doi: 10.1073/pnas.1112 378108

Reyes-Haro, D., Müller, J., Boresch, M., Pivneva, T., Benedetti, B., Scheller, A., et al. (2010). Neuron-astrocyte interactions in the medial nucleus of the trapezoid body. J. Gen. Physiol. 135, 583-594. doi: 10.1085/jgp.200910354

Rosenberg, P. A., Knowles, R., Knowles, K. P., and Li, Y. (1994). Beta-adrenergic receptor-mediated regulation of extracellular adenosine in cerebral cortex in culture. J. Neurosci. 14(5 Pt 2), 2953-2965.

Sasaki, T., Beppu, K., Tanaka, K. F., Fukazawa, Y., Shigemoto, R., and Matsui, K. (2012). Application of an optogenetic byway for perturbing neuronal activity via glial photostimulation. Proc. Natl. Acad. Sci. U.S.A. 109, 20720-20725. doi: 10.1073/pnas. 1213458109

Seifert, G., and Steinhäuser, C. (2013). Neuron-astrocyte signaling and epilepsy. Exp. Neurol. 244, 4-10. doi: 10.1016/j.expneurol.2011.08.024

Semyanov, A., Walker, M. C., Kullmann, D. M., and Silver, R. A. (2004). Tonically active $\mathrm{GABA}_{A}$ receptors: modulating gain and maintaining the tone. Trends Neurosci. 27, 262-269. doi: 10.1016/j.tins.2004.03.005

Shan, D., Yates, S., Roberts, R. C., and McCullumsmith, R. E. (2012). Update on the neurobiology of schizophrenia: a role for extracellular microdomains. Minerva Psichiatr. 53, 233-249.

Shigetomi, E., Bowser, D. N., Sofroniew, M. V., and Khakh, B. S. (2008). Two forms of astrocyte calcium excitability have distinct effects on NMDA receptor-mediated slow inward currents in pyramidal neurons. J. Neurosci. 28, 6659-6663. doi: 10.1523/JNEUROSCI.1717-08.2008

Sica, R. E. (2015). Could astrocytes be the primary target of an offending agent causing the primary degenerative diseases of the human central nervous system? A hypothesis. Med. Hypotheses 84, 481-489. doi: 10.1016/j.mehy.2015.02.004

Szatkowski, M., Barbour, B., and Attwell, D. (1990). Non-vesicular release of glutamate from glial cells by reversed electrogenic glutamate uptake. Nature 348, 443-446. doi: 10.1038/348443a0

Talantova, M., Sanz-Blasco, S., Zhang, X., Xia, P., Akhtar, M. W., Okamoto, S., et al. (2013). A $\beta$ induces astrocytic glutamate release, extrasynaptic NMDA receptor activation, and synaptic loss. Proc. Natl. Acad. Sci. U.S.A. 110, E2518-E227. doi: 10.1073/pnas. 1306832110

Tian, G. F., Azmi, H., Takano, T., Xu, Q., Peng, W., Lin, J., et al. (2005). An astrocytic basis of epilepsy. Nat. Med. 11, 973-981. doi: 10.1038/ nm 1277

Verkhratsky, A., Rodríguez, J. J., and Parpura, V. (2012). Calcium signalling in astroglia. Mol. Cell. Endocrinol. 353, 45-56. doi: 10.1016/j.mce.2011. 08.039

Wang, C. M., Chang, Y. Y., Kuo, J. S., and Sun, S. H. (2002). Activation of P2X(7) receptors induced $[(3) \mathrm{H}] \mathrm{GABA}$ release from the RBA-2 type-2 astrocyte cell line through a $\mathrm{Cl}(-) / \mathrm{HCO}(3)(-)$-dependent mechanism. Glia 37, 8-18. doi: 10.1002/glia.10004

Warr, O., Takahashi, M., and Attwell, D. (1999). Modulation of extracellular glutamate concentration in rat brain slices by cystine-glutamate exchange. J. Physiol. 514(Pt 3), 783-793. doi: 10.1111/j.1469-7793.1999. 783ad.x

Ye, Z. C., Wyeth, M. S., Baltan-Tekkok, S., and Ransom, B. R. (2003). Functional hemichannels in astrocytes: a novel mechanism of glutamate release. J. Neurosci. 23, 3588-3596.

Zhou, Y., and Danbolt, N. C. (2013). GABA and glutamate transporters in brain. Front. Endocrinol. 4:165. doi: 10.3389/fendo.2013.00165

Zimmer, E. R., Torrez, V. R., Kalinine, E., Augustin, M. C., Zenki, K. C., Almeida, R. F., et al. (2015). Long-term NMDAR antagonism correlates reduced astrocytic glutamate uptake with anxiety-like phenotype. Front. Cell. Neurosci. 9:219. doi: 10.3389 /fncel.2015.00219

Conflict of Interest Statement: The author declares that the research was conducted in the absence of any commercial or financial relationships that could be construed as a potential conflict of interest.

Copyright (c) 2015 Pál. This is an open-access article distributed under the terms of the Creative Commons Attribution License (CC BY). The use, distribution or reproduction in other forums is permitted, provided the original author (s) or licensor are credited and that the original publication in this journal is cited, in accordance with accepted academic practice. No use, distribution or reproduction is permitted which does not comply with these terms. 